\title{
The method of adjustment of heat supply systems with the multistage temperature control at pumping stations
}

\author{
Vyacheslav Tokarev ${ }^{1, *}$, and Nikolay Novitsky ${ }^{1}$ \\ ${ }^{1}$ Melentiev Energy Systems Institute of the Siberian Branch of the Russian Academy of Sciences, \\ 664033, Lermontova str., 130, Irkutsk, Russia
}

\begin{abstract}
The lowering of the temperature graph in the lower-level heat networks is often carried out by a mixture of coolant from the return pipeline at mixing pumping stations in order to maintain the set temperature of the mixed water. In this case, part of the heat carrier is not returned to the source of thermal energy, but it circulates in the circuits from the mixing station to the lower consumption nodes. The presence of such circulation flows leads to the absence of a stabilizing moment of the temperature field when it is calculated by traditional methods, as well as to the "looping" of the computational process and the impossibility of obtaining a solution. In work to overcome these problems, a new computational scheme for commissioning calculation is proposed, which is based on: (a) decomposing the calculation of the thermal-hydraulic regime into calculations of the hydraulic and temperature conditions; (b) using the conditional regulator of the ratio of the mixing station's output costs in calculating the hydraulic regime; (c) fixing the temperature at the station's output when calculating the temperature regime; (d) iterative calculation with correction of the setpoint of the ratio controller of the flow rate based on the calculation of the temperature regime.
\end{abstract}

\section{Introduction}

Now schemes of many heat supply systems (HSS) of large cities include such control elements as mixing pump stations (MPS). These elements are used to lower the temperature in the supply line due to the addition of water from the return pipeline. In this case, there is a transition from one temperature graph (TG) of qualitative regulation to another (lowered). The need for such a transition is dictated by the type of heat insulation of the pipelines, which is sometimes not designed for high temperatures, and/or the scheme for connecting heat consumers to the heat network (HN). MPS are common in systems that have many compactly located consumers with direct connection to the $\mathrm{HN}$ and low heat load, when it is impossible to organize elevator water mixing at the consumer due to low flow of coolant or insufficient available pressure. Also, the use of MPS allows raising the temperature of the coolant in the supply pipeline to the MPS (switch to another TG) in order to increase the

* Corresponding author: tslava@ isem.irk.ru 
throughput capacity of the main section of the transit pipeline.

A number of cities use a multistage cascade change in TG. For example, the coolant is released from the source according to the schedule 170/70 and is transmitted by transit to the entrance of the city, where drops to $150 /$ due to the MPS the TG 70 . Further, the temperature is reduced to $130 / 70$ by the following MPS in front of the HN zone with the thermal insulation capable of withstanding a temperature of no higher than $130^{\circ} \mathrm{C}$. In front of a group of directly connected consumers, the return water is mixed with water in the feed for the third time to go to TG 95/70. Approximately such a scheme of using the MPS is used in the HSS of Irkutsk (Figure 1), which includes 27 MPS.

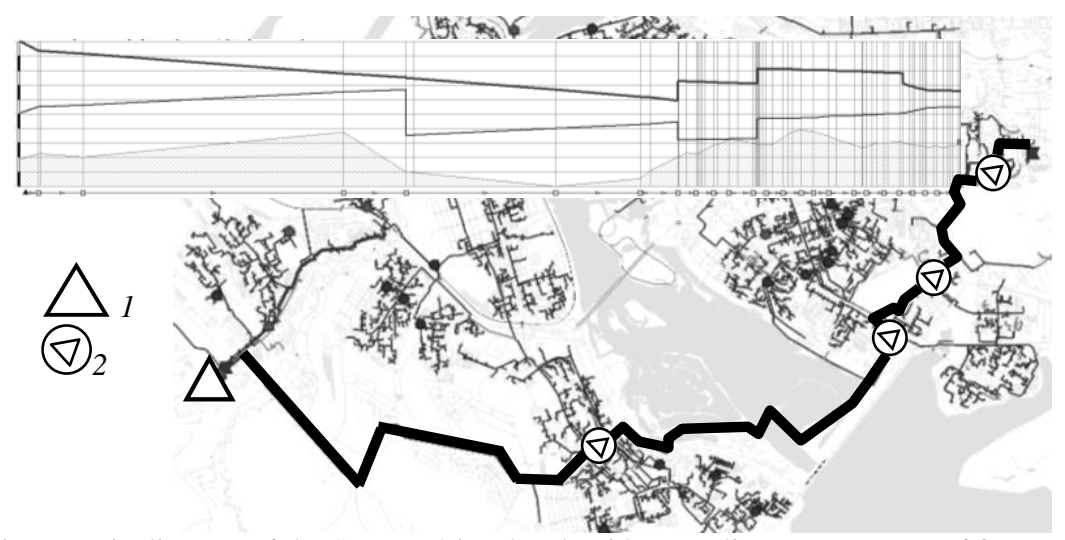

Fig. 1. Piezometric diagram of the SH No. 4 in Irkutsk with cascading arrangement of four pumping mixing stations. 1: source of heat (Novo-Irkutsk CP); 2: the mixing pump station.

Since the MPS does not require the installation of heaters, and the flow of mixed water is much less than the circulating water, the cost of the MPS device will be much lower than when connecting the distribution networks to the mains in the central heating station (CHS) in an independent scheme. However, in the MPS, the hydraulic mode of the distribution networks depends on the mode in the backbone network, and the possible range of regulation of the heads is less than in the HSS with independent connection through the CHS. The installation of pumps in the MPS is not on the jumper but on the return pipeline up to (along the coolant path) the mixing jumper (together with an uncomplicated automation circuit) allows not only to provide a return water pile, but also to reduce the pressure in the return pipeline for consumers after the MPS, although it requires installation of larger pumps. All this determines the complexity, relevance, and practical significance of the task of calculating the regimes with the MPS.

\section{Analysis of the existing approaches to the modeling of HSS modes with mixing pumping stations}

When controlling the HSS mode, the value of the water admixture from the return pipeline maintains the temperature of the mixed water at the outlet of the MPS for consumers in the supply zone. In this case, part of the coolant, not reaching the sources of heat energy ( $\mathrm{SH})$, returns to consumers (Figure 2). The constantly circulating (cooling) part of the flow, not heated by $\mathrm{SH}$, leads to the absence of the moment of the temperature field stabilization when it is calculated by traditional methods, to the "looping" of the computational process, the impossibility of obtaining a solution. 


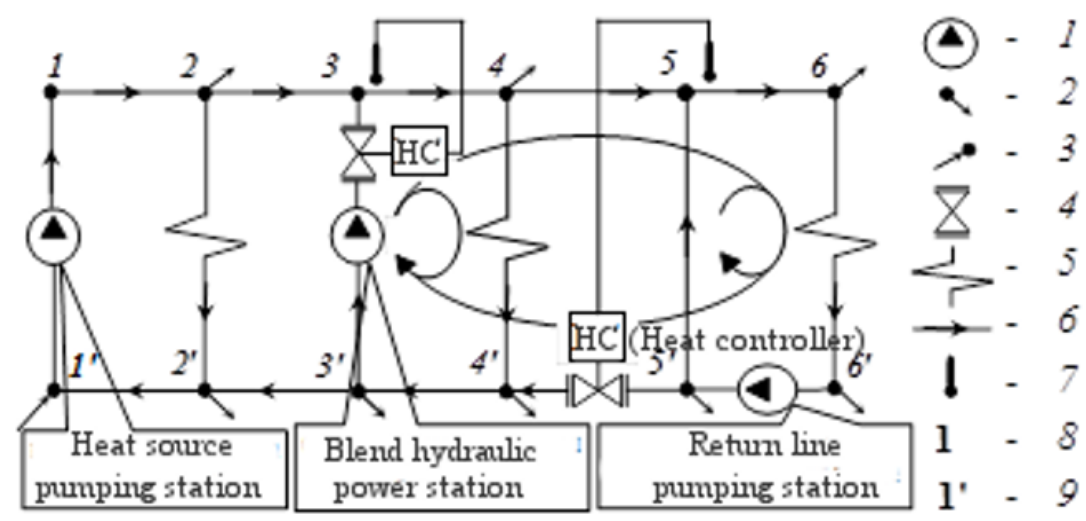

Fig. 2. Type of the design scheme with mixing pumping stations (arrows in the diagram show the nested loops of the closed circulation), where: 1 - source of pressure; 2 - withdrawal of water from the unit (for hot water supply); 3 - the inflow of water into the unit; 4 - the regulating valve; 5 - a local heating and ventilation system; 6 - a pipeline section (the arrow indicates flow direction); 7 control point for the mixed water temperature controller; 8 - the number of the node on the supply line; 9 - the number of the node on the return pipeline; MTR (HC) - mixing temperature regulators.

Studies show that in practice design and operation is widely used a technique associated with the artificial rupture of the HSS scheme before the MPS and the subsequent linkage of the boundary parameters at the point of rupture, which is often not automated and carried out by an expert engineer. The required calculation time increases very significantly, and the solution cannot be obtained in some cases, for example, if the MPS is installed on a ring network in a HSS with several sources.

\section{Methodology}

On the basis of the scientific direction developed and developed in the MESI SB RAS, the theory of hydraulic circuits (THCs) [1, 9], a unique experience in creating methodical software for solving the problems of calculating and optimizing pipeline systems of various types and purposes [3-8] in dozens of research, design, and operational enterprises of the country, as well as abroad. In particular, on this basis, the information and computer complex (ICC) "ANGARA-TS" has been developed, which provides the possibility of solving routine and setup problems for networks of arbitrary configurations (branched, multi-loop) and structures (with arbitrary number and location of pumping stations, sources, regulators of pressure or the expense), connecting the consumers of heat having heterogeneous loads. The ICC also has high speed, an average of 20-50 seconds. It also provides an opportunity for multi-loop networks containing about 25000 branches of the design scheme and allows one- and multi-level calculations. The HSS multilevel modeling, as a scientific and methodological approach, is designed to overcome the problems of great complexity and dimension of both HSS itself and the problems arising here $[10,11]$. To take into account the network specificity of the problems of calculating the thermalhydraulic HSS modes [1,9], the graph theory is used as a visual and effective section of mathematics from the point of view of algorithmization and software implementation. The basic methods for calculating hydraulic regimes are the general methods for calculating the flow distribution: the method of nodal pressures [12], the contour flow method [1], the global gradient method [13], and other methods [14-18].

The basic for solving the problems of calculation and analysis of the HSS modes is the model of the thermohydraulic regime (THR) in nodal form [9]. At the same time, the 
possibility of decomposition of the thermohydraulic model into the model of hydraulic (GR) and thermal regimes (TP) is widely used $[5,10]$. To obtain a solution, it suffices to solve successively the problems of calculating the GR and TP [19].

Among the main methods of calculating the GR in networks equipped with regulators, two approaches dominate. The first one is connected with applying the technique of "double" (or nested) iteration cycles [1]. At the same time, the internal task solves the task of stream distribution with fixed parameters of the mode being supported by regulators, while he external one corrects the parameters of the regulating devices. The second [20] is based on the "convolution-scan" technique, which consists in adjusting a mathematical model of the network on the external cycle of iterations, depending on the state of the regulators. In [12], the "relay" method was proposed for the first time, which makes it possible to significantly accelerate the computation by eliminating the outer iteration cycles by changing the TPS model on the internal iteration cycles, depending on the state of the control elements (regulators). In [8] a "relay" method for calculating the GR HSS with cost ratio regulators (CRR) was developed.

\section{Statement of the problem of the adjustment thermohydraulic calculation and its modification}

The task of adjustment calculation of the THR HNC is solved with the aim of determining the parameters of the throttling devices (regulators) that provide the required heat costs to consumers with given restrictions on the parameters of the regime [19].

Since the water flow through the MPS depends on the temperature of the mixed water, and it, in turn, depends on the flow distribution and the heat losses in the network, it is not possible to determine the flow distribution for one calculation of GR and TP. Therefore, an external iteration cycle is required, the stopping criterion in which will be achieving of required mixed water temperature $t_{3}$ with a specified accuracy. In addition, special methods and stopping criteria must be applied to calculate the temperature field in the TS. This is due to the fact that contours of closed circulation appear in the network, in the case of cascade mixing these circuits are nested (see Figire 2).

As a result of the GR calculation at fixed temperatures (at the $\mathrm{k}_{\text {th }}$ iteration of the outer cycle of temperatures at the HSS nodes), the flow of admixed water $\left(x_{2}^{(k)}\right)$ passing through the MPS (Figure 3) is uniquely determined by the flow of network water in the supply line to the MPS $\left(x_{1}^{(k)}\right)$ and the mixing ratio $(U)$ :

$$
U=\left(t_{1}-t_{3}\right) /\left(t_{3}-t_{2}\right),
$$

where $t_{2}, t_{2}$ and $t_{3}$ are, respectively, the water temperatures in the supply line to the MPS, in the return line, and the required temperature of the mixed water in the supply line after the MPS. 

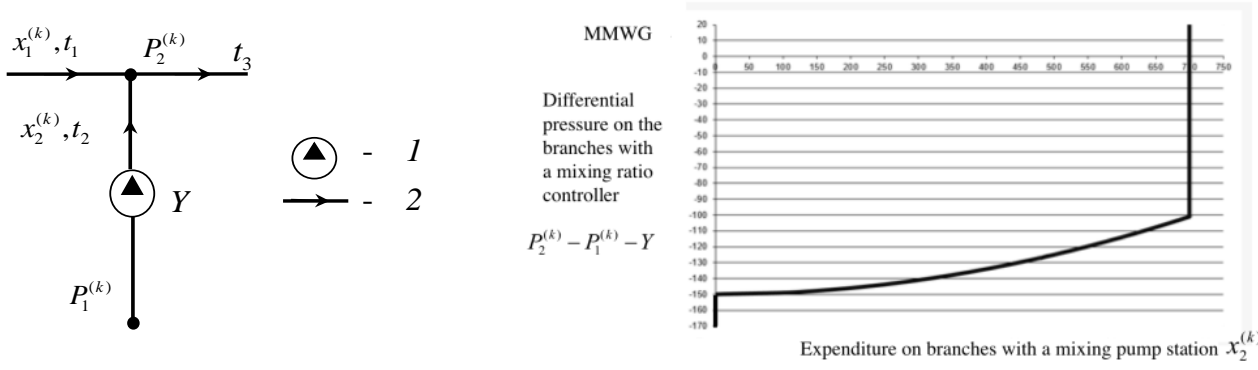

Fig. 3. A mixing unit with the ratio controller of the flow rate and its hydraulic characteristic at the kth iteration of the external cycle flow rate known $x_{1}^{(k)} ; U x_{1}^{(k)}=700 \mathrm{t} / \mathrm{h}$ and $Y=150 \mathrm{mmwg}: 1-$ the source of pressure; 2 - a pipeline section (arrows indicate flow directions).

Thus, in the case of GH, the section with the MPS can be considered as a section with an active acting head and temperature controlled mixed water CRR at the end of this section.

The meaningful formulation of the task of the adjustment GR is as follows. Given: the design scheme (see Figure 2), which uniquely determines the matrix $A$; hydraulic characteristics for all its $n$ branches, $f(x)$; costs in $m$-1 nodes (vector $Q$ ); a vector of the known pressure increments for active elements (active heads at sources, NS and MPS); mixing coefficients on the MPS (vector $U$ ); pressure in one of the nodes $\left(P_{m}^{\prime}\right)$. It is required to determine the costs (vector $x$ ) and pressure drops (vector $y$ ) across all branches, as well as node pressures in $m-1$ nodes (vector $P$ ) that satisfy the MPS operation conditions.

\section{Method for solving the task of calculating the thermal- hydraulic regime}

With the adjustment of the THR, the mixing node with the MPS is proposed to be considered as a water temperature controller by mixing, and the temperature at the outlet of the MPS should be fixed at the level required by the TG. In this case, the task of adjusting the thermal and hydraulic calculation is to determine the flow distribution in the network to ensure the required loads of consumers, including the amount of water flow mixed from the return pipeline to the MPS to ensure the set temperature of the mixed water. The proposed new methodology is based on the combination of the following stages of calculation: 1) decomposition of the calculation of the thermal-hydraulic regime for the GR and TP calculations; 2) the use of a conditional POR with a given mixing ratio of $U$ fluxes at the outlet of the MPS when calculating the hydraulic regime; 3 ) fixing the temperature $t_{3}$ at the outlet of the MPS during the calculation of the temperature regime; 4) correction of the CRR setpoint $\left(U^{*}\right)$ according to the results of the calculation of the temperature regime; 5) iterative execution of par. 2.-4. until reaching the stabilization of the flow of admixed water $\left(x_{2}^{(k)}\right)$ with a specified accuracy.

The proposed approach makes it possible to determine the TGS HSS from the MPS without using decomposition techniques for the network design scheme with subsequent laborious linking of the boundary parameters at the decomposition points.

\section{Conclusion}

The proposed methodology was implemented as part of the "ANGARA-TS" test system and tested on test and real-life examples of large-scale thermal networks that confirmed its 
efficiency (the THR calculation stably converges for several external iterations). Automation of the accounting of the RFP in the CPI with the help of the "relay" method in the HSS calculation in Irkutsk resulted in a reduction in the number of GR iterations from 80 to 60 and allowed to avoid an external iteration cycle for adjusting the settings of the flow regulator previously used in modeling the HSS that contained the MPS.

The research was carried out within the framework of the project of the fundamental research program of the Siberian Branch of the Russian Academy of Sciences (AAAA-A17117030310437-4) III.17.4.3, with the financial support coming from the Russian Foundation for Basic Research and the Government of the Irkutsk Region within the framework of the scientific project No. 17-48-380021.

\section{References}

1. Theory of hydraulic circuits (Science, Moscow, 1985)

2. Mathematical modeling and optimization of heat, water, oil and gas supply systems (Nauka, Novosibirsk, 1992)

3. Hydraulic chains: the development of theory and applications (Nauka, Novosibirsk, 2000)

4. Pipeline systems of power engineering: developing the methods of mathematical modeling and optimization (Nauka, Novosibirsk, 2008)

5. Pipeline energy systems: mathematical modeling and optimization (Nauka, Novosibirsk, 2010)

6. Pipeline energy systems: mathematical and computer modeling (Nauka, Novosibirsk, 2014)

7. Pipeline systems of power engineering: methodical and applied problems of mathematical modeling (Nauka, Novosibirsk, 2015)

8. Pipeline energy systems: mathematical and computer technologies of intellectualization (Nauka, Novosibirsk, 2017)

9. Z. I. Shalaginova, Thermal Engineering, 12, 1016 (2009)

10. N. N. Novitsky, Z. I. Shalaginova, V. V. Tokarev, O. A. Grebneva, Proceedings of the Russian Academy of Sciences - Power Engineering, 1, 12 (2018)

11. N. N. Novitsky, A. V. Alekseev, O. A. Grebneva, A. V. Lutsenko, V. V. Tokarev, Z. I. Shalaginova. Energy (https://doi.org/10.1016/j.energy.2018.02.070, 2018)

12. N. N. Novitsky, V. V. Tokarev, Proceedings of the Russian Academy of Sciences Power Engineering, 2, 88 (2001)

13. E. Todini , S. Pilati , Comp. Appl. in Water Supply, 1, 1 (1988)

14. J. Ortiga, J. C. Bruno, A. Coronas, I. E. Grossman, ESCAPE17 (2007, Elsevier)

15. A. A. Filimonova, T. A. Barbasova, D. A. Shnayder, A. A. Basalaev, En. Proc, 111, 710 (2017)

16. M. Vesterlund, J. Dahl, En. Conv. and Management. 89, 555 (2015)

17. E. Guelpa, C. Toro, A. Sciacovelli, R. Melli, E. Sciubba, V. Verda, Energy, 102, 586 (2016)

18. G. Schweiger, P.-O. Larsson, F. Magnusson, P. Lauenburg, S. Velut, Energy, 137, 566 (2017)

19. V. V. Tokarev, Z. I. Shalaginova, Thermal Engineering, 1, 68 (2016) 
20. Mathematical modeling and optimization of developing heat supply systems (Nauka, Novosibirsk, 1987) 